\title{
Gingival hyperplasia as an emanation of wire and braces: A case report
}

\author{
Shivani Jain ${ }^{1}$, Gouri Bhatia ${ }^{2}$, Deepa $\mathrm{D}^{3^{*}}$ \\ ${ }^{1} \mathrm{PG} 3^{\text {rd }}$ Year, ${ }^{2}$ Reader, ${ }^{3}$ Professor and Head, Dept. of Periodontology, Teerthanker Mahaveer Dental College \& Research Centre, \\ Moradabad, Uttar Pradesh, India \\ *Corresponding Author: Deepa D \\ Email: deepa_arun@rediffmail.com
}

\begin{abstract}
Gingival hyperplasia, hypertrophy \& gingival overgrowth are all synonymous terms for the abnormal overgrowth of gingival tissues. A male patient aged 19 years presented with chronic inflammatory gingival enlargement in upper \& lower arch associated with prolonged orthodontic therapy. Surgical therapy was performed after scaling and root planing to provide a good aesthetic outcome. Two different surgical treatment modalities were performed in this case, one by using scalpel and second by electrosurgery unit which were compared to evaluate the effect on wound healing \& tissue handling properties. Even though scalpel remains a gold standard, electrosurgery showed advantageous over the scalpel for treatment of gingival hyperplasia associated with orthodontic patients.
\end{abstract}

Keywords: Aesthetics, Gingival hyperplasia, Electrosurgery, Non surgical, Surgical, Scaling root planing.

\section{Introduction}

Gingival hyperplasia or gingival overgrowth is defined as increase in the size of the gingiva $\&$ is considered as a feature of diseased gingival tissue. It can be fibrous or inflammatory, mainly dependent on the etiological factor. It may be associated with accumulation of plaque with multiple species of microorganisms \& hormonal imbalance due to host microbial interaction to various stimuli. ${ }^{1}$

Gingival overgrowth can also be an expression of numerous blood disorders, for example leukemia, thrombocytopenia etc. A very rare variant of gingival enlargement i.e. idiopathic gingival fibromatosis, has been found to be hereditary disease with familial inheritance. An inflammatory response in gingiva has also been observed in Type IV hypersensitivity with orthodontic wires containing nickel which ultimately leads to nickel allergic contact stomatitis but its etiology has not yet clearly been defined (Holmstrup, 1999; Vanarsdall, 2000). Eventually overgrowth may result into few problems like altered speech, poor aesthetics \& difficulty in chewing and psychosomatic problems in the individuals suffering from it. $^{2}$

There are two phases of inflammatory gingival enlargement, acute phase or chronic phase \& alterations in the gingival tissue can easily be recognized in chronic phase. In fixed orthodontic appliances patient's proper oral hygiene maintenance becomes difficult \& these fixed appliances act as retention areas for accumulation of debris and plaque which may further worsen the condition. ${ }^{1}$

Different treatment modalities have been proposed for the management of gingival overgrowth which include non surgical, surgical and pharmacological management. Sometimes non surgical treatment alone is not sufficient for complete resolution of the problem hence surgical therapy and pharmacological intervention may be required. Surgical therapy incorporates excision of gingival overgrowth with conventional technique using scalpel. ${ }^{3}$

However, there are certain disadvantages of using scalpel blade which were resolved by the evolution of electrosurgery \& has been used since 1928 in dentistry for soft tissue procedures like gingivectomy, gingivoplasty, soft tissue growth excision, crown lengthening etc.

Therefore, the aim of this case report was to evaluate and compare the healing after excision of gingival overgrowth using scalpel blade and electrocautery in orthodontic patient.

\section{Case Report}

A 19 year old systemically healthy male patient reported to our department of periodontology of Teerthanker Mahaveer dental college and research centre, Moradabad, Uttar Pradesh, with the chief complaint of swelling in gums in upper and lower front teeth region since 1month. Patient was apparently asymptomatic 1 month back suddenly he noticed gingival swelling in upper and lower front teeth region which gradually increased in size mainly in the interdental area. Patient gave history of undergoing orthodontic treatment for last 1 year. Intraoral examination revealed inflammatory enlargement with respect to 13-23 and 33-43 (Fig. 1).

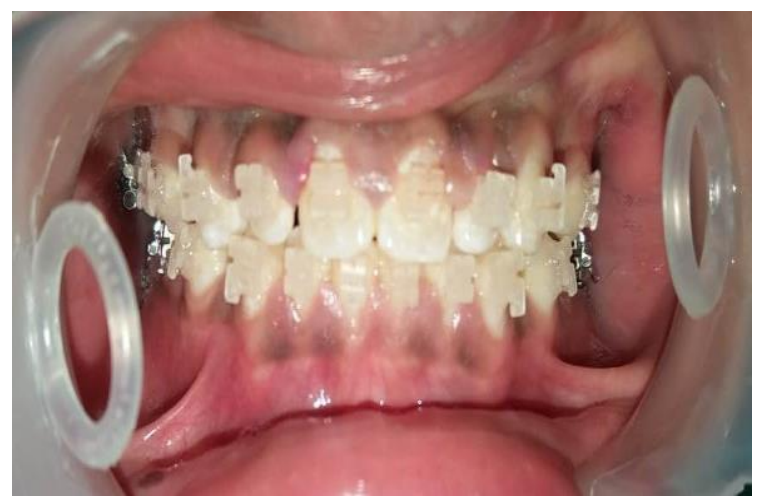

Fig. 1: Preoperative

Excision of enlarged gingival tissue was planned with respect to 13-23 \& 33-43 after scaling and root planing. Patient was informed about the treatment, blood 
investigations were done and written consent was taken from the patient before initiation of the treatment during the first visit. During the surgical appointment, local anaesthesia was infiltrated in the area of surgery, pseudo pockets were marked and measured \& external bevel incision was given at 1.5-2 mm distance from gingival margin of with the help of 15 no. scalpel blade with respect to $13-23$ to excise the enlarged tissue following the scalloping of the gingival margin (Fig. 2, Fig. 3). Gingival collar which was formed after these incisions was removed with the help of curette (Fig. 4).

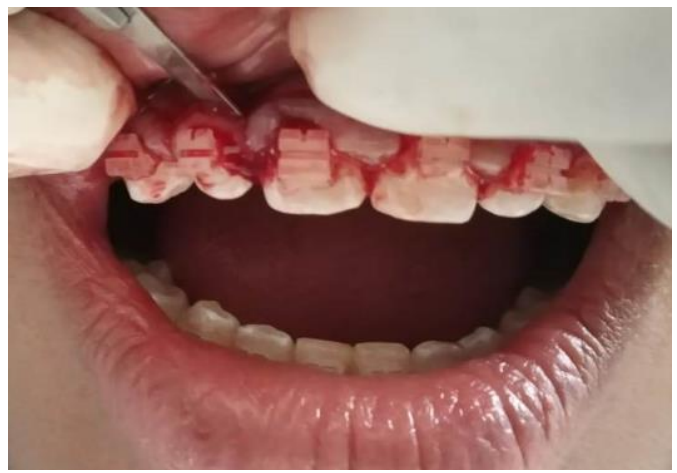

Fig. 2: Excision of gingival tissue using scalpel

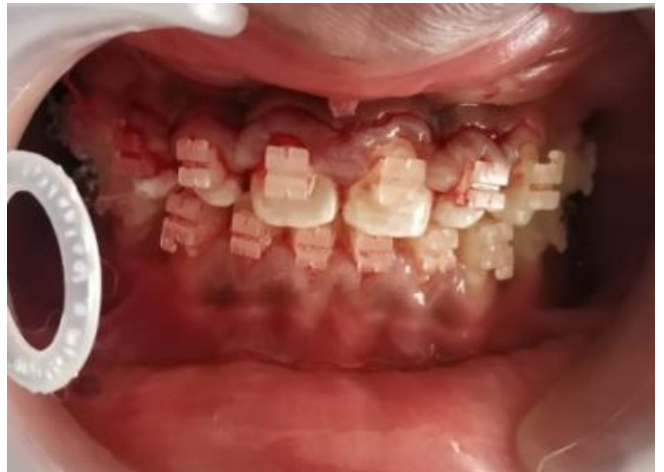

Fig. 3: Intra-operative

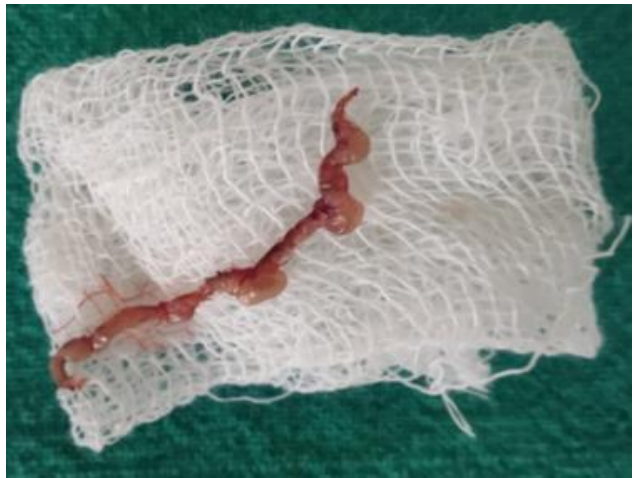

Fig. 4: Excised tissue

Subsequently, similarly in lower arch from 33-43, enlarged gingival tissue was excised with the help of electrocautery simultaneously to compare the effect of scalpel blade and electrocautery on healing of soft tissue (Fig. 5-7).

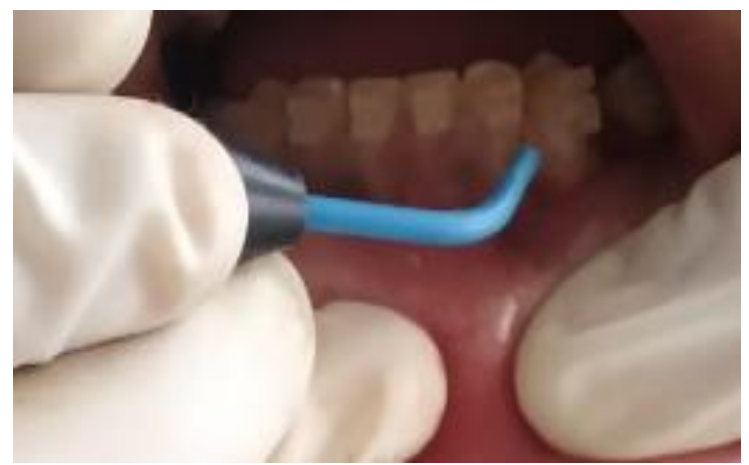

Fig. 5: Excision of gingival tissue using electro cautery

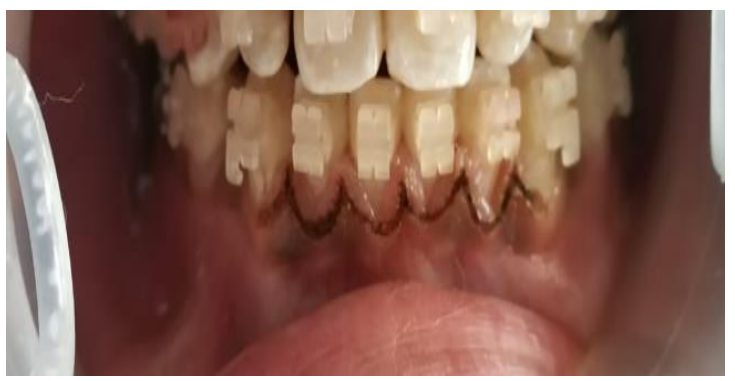

Fig. 6: Intraoperative

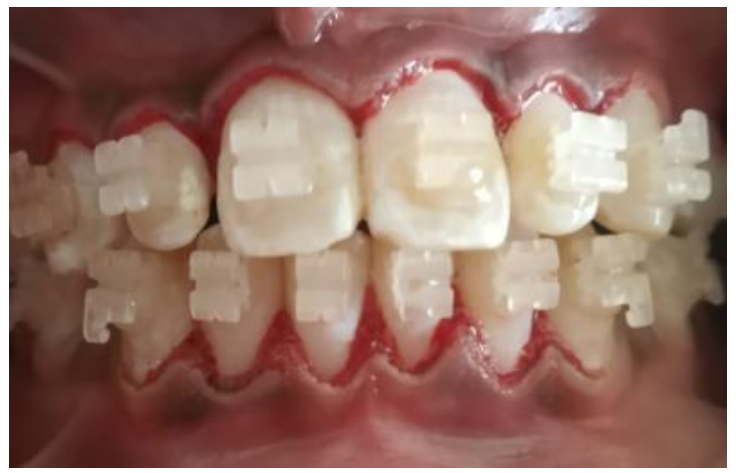

Fig. 7: Postoperative

Post-operative oral hygiene instructions was given and analgesics (diclofenac sodium +paracetamol) was prescribed for 3 days and recalled after 10 days for reevaluation of soft tissue healing (Fig. 8).

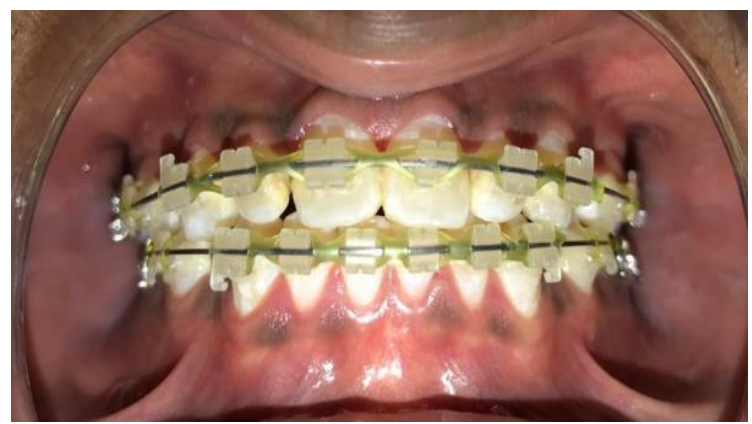

Fig. 8: After 10 days 


\section{Discussion}

Most common cause for chronic inflammatory gingival enlargement is prolonged exposure to plaque which leads to infective cellular infiltration \& clinically appears as soft and edematous gingiva which can be treated by conventional periodontal treatment, including regular professional oral prophylaxis and good patient compliance. As in all cases, patient education, motivation and compliance during and after dental treatment is most important. ${ }^{1}$ Reinforcement of oral hygiene is necessary as there is tendency to revert. Current case shows that enlargement of gingival tissues was not due to patient negligence in maintaining proper oral hygiene but it was due to the fixed appliances which were acting as retention areas for accumulation of plaque \& also acting as hindrance in maintaining healthy oral hygiene. ${ }^{1}$

Improvement of oral hygiene maintenance could be carried out by many methods however the traditional method of removing plaque by tooth brushing is the most efficient mechanical method. End-tufted brushes along with disinfectants such as chlorhexidine, supplemented with floss threaders or stiff plastic floss that can be threaded beneath the arch wires, are mostly useful for orthodontic patients. However, time ranges from 15 to 30 minutes daily required for effective plaque control. ${ }^{2}$

Here, we report a case of gingival hyperplasia associated with orthodontic treatment in which enlargement of gingival tissues are mainly due to the microbial deposits i.e. plaque and calculus. Following orthodontic treatment gingival hyperplasia is very common compared to other oral manifestations (Genelhu et al, 2005; Kouraki et al., 2005). Enlargement associated with orthodontic appliance is mostly fibrotic which differs from allergic or inflammatory gingival lesions with fragile and reddened gingival (Zachrisson and Zachrisson, 1972; Ramadan, 2004). However, there is no clear definition on its initiation and histopathology. ${ }^{3}$

A study by Sallum et al \& Carranza 1996 showed that removal of fixed orthodontic appliance and subsequent oral prophylaxis can revert back the gingival tissues to its healthy state. ${ }^{2}$ However, it was concluded that complete resolution of fibrous gingival enlargement is not possible without surgical intervention by Ramadan in 2004 .

As professional prophylaxis alone cannot resolve the gingival hyperplasia associated with orthodontic appliance completely, therefore various other treatment modalities have been proposed for complete resolution of the condition which includes surgical intervention \& pharmacological therapy along with professional oral prophylaxis. Surgical intervention includes excision of soft tissue overgrowth with the help of scalpel (conventional technique). But the main disadvantages of using scalpel are inadequate visibility, excessive bleeding \& painful for the patient so to overcome the limitations of conventional technique, with the advent of medical science \& technology newer technique such as electrosurgery has been gaining attention with its advantages like ease of use, providing almost clear or bloodless operating field with immediate haemostasis, more comfortable to the patient \& completed in shorter duration as compared to conventional procedure. ${ }^{4}$

In the present case, after completing both the treatment modalities, oral hygiene instruction was given to the patient and patient was motivated $\&$ educated about the importance of maintaining a healthy oral environment $\&$ the negative effects of poor oral hygiene on gingival tissue. ${ }^{4}$

Various chemical plaque control methods have been used in patients undergoing orthodontic therapy. In various studies use of chlorhexidine has shown conflicting results on the efficacy with some studies reporting favorable results while others reporting unfavorable results. A study conducted by Anderson et al evaluated recently the role of chlorhexidine \& concluded that it was a valuable aid in reduction of plaque $\&$ subsequently gingivitis when used as an adjunct to regular oral hygiene practices. ${ }^{5}$

Since the primary aim of any therapy is to re-establish the aesthetic and function, surgical therapy becomes the treatment of choice. ${ }^{6}$ In the present case report postoperative healing was observed to be satisfactory with both conventional procedure as well as with electrosurgery unit. Although electrosurgery is an advanced treatment modality with clear advantage over the scalpel in terms of reduced procedure time and immediate haemostasis they have some distinct disadvantages also namely lateral heat damage and prolonged tissue healing. Conventional scalpel therapy provides precise incision line \& faster healing at much lower cost. ${ }^{7}$ Even though scalpel remains a gold standard, electrosurgery showed advantageous over the scalpel for treatment of gingival hyperplasia associated with orthodontic patients.

\section{Conclusion}

Successful treatment of gingival hyperplasia an emanation of wire and braces depends on the proper recognition of causative factors and by improving oral hygiene status, aesthetics \& function via removal of local irritating factors and surgical excision of the over growth. However there are chances of recurrence, if patient ignores oral hygiene routine following surgical treatment.

\section{Source of funding}

None.

\section{Conflict of interest}

None.

\section{References}

1. Tuli AM, Bhatnagar N. Gingival hyperplasia a sequalae of orthodontic therapy- A Case Report. Acta Scientific Dental Sci 2018;3(1):84-86.

2. Jadhav T, Bhat KM, Bhat GS, Varghese JM. Chronic inflammatory gingival enlargement associated with orthodontic therapy - A case report. J Dent Hyg 2013;87(1):19-23.

3. Gursoy UK, Sokucu O, Uitto VJ, Aydin A, Demirer S, Toker $\mathrm{H}$ et al. The role of nickel accumulation and epithelial cell proliferation in orthodontic treatment-induced gingival overgrowth. Eur J Orthodont 2007:555-8. 
4. Krishnan V, Ambili R, Davidovitch Z, Murphy NC. Gingiva and orthodontic treatment. Semin Orthod 2007;13(4):257-71.

5. Chabria D, Weintraub RG, Kilpatrick NM. Mechanisms and management of gingival overgrowth in pediatric transplant recipients: a review. Int J Paediatr Dent 2003;13(4):220-9.

6. Shafi M, Khan N, Gupta P, Mir RA, Bashir N. Surgical management of gingival enlargement: a case series. Int J Cur Res Rev 2014;6(15):53-6.

7. Baburaj FS, Pimpale SK. Comparison between laser, electrocautery and scalpel in the treatment of drug-induced gingival overgrowth: a case report \& reviews. Int J Sci Study 2015;1(10):27-30.

How to cite: Jain S, Bhatia G, Deepa D. Gingival hyperplasia as an emanation of wire and braces: A case report. IP Int J Periodontol Implantol 2020;5(1):33-6. 